\title{
Cervical Spondylotic Myelopathy Mimicking Amyotrophic Lateral Sclerosis
}

\author{
Halil Ondera, b, F. Gokcem Yildiz ${ }^{\mathrm{a}}$
}

\section{To the Editor}

A 53-year-old man was admitted to our clinic with weakness in all extremities, predominantly in upper limbs which had started 2 years ago asymmetrically and gradually progressed. He had been working as a furniture transporter for many years and he was unable to work for the last 6 months due to the severe weakness. Upon history taking, we learned that he had been suffering only very slightly from neck and back pains and noticed widespread but mild fasciculation for a while. On neurological examination, upper extremity muscle strength was evaluated as $2 / 5$ in proximal muscles and $3+/ 5$ in distal muscles (Medical Research Council scale). Lower extremity proximal and distal muscle strengths were evaluated as $4+/ 5$ bilaterally. Deep tendon reflexes were hyperactive prominently in lower limbs. Not any sensorial deficit was found. Hence, considering the pre-diagnosis of amyotrophic lateral sclerosis (ALS), he was consulted to electromyography (EMG) laboratory. Motor and sensory nerve conduction studies were in normal range in all the extremities. Needle EMG revealed widespread fasciculation and subacute denervation changes were seen in cervical and lumbar myotomes, which were significant in bilateral C4-6 segments. On the other hand, further needle EMG examinations of paraspinal muscles, transverse abdominis and genioglossus were found to be normal. EMG results were atypical for ALS and predominance of denervation in upper extremities was suggestive of an underlying cord pathology. Hence, spinal MRI was performed which showed disc protrusions in multiple segments and a posterior disc hernia between $\mathrm{C} 5$ and 6 segments, resulting in longitudinal myelitis spreading two segments both upper and lower of the spine (Fig. 1). As a result of imaging and electromyography findings, we made the diagnosis of multiple disc pathologies and seconder myelitis and consulted the patient to neurosurgery department. Follow-up evaluation after surgery was also planned in the outpatient clinic.

ALS is a progressive neurodegenerative disorder affecting

Manuscript accepted for publication August 09, 2016

${ }^{a}$ Neurology Department, Hacettepe University Hospital, Sihhiye, Ankara, Turkey

${ }^{\mathrm{b}}$ Corresponding Author: Halil Onder, Neurology Department, Hacettepe University, Sihhiye, Ankara, Turkey. Email: halilnder@yahoo.com

doi: http://dx.doi.org/10.14740/jnr392e upper and lower motor neurons and with a fatal outcome [1]. However, cervical spondylotic myelopathy, which has a better prognosis and can be improved by surgery, may mimic ALS. Hence, differentiation of these entities in the early phase constitutes a crucial deal for clinicians as it may sometimes be quite challenging $[1,2]$. On the other hand, degenerative changes of spine frequently may also coexist in elderly patients with ALS which makes the differential diagnosis furtherly hard [1]. Here, we present a case at whom via combined neuroimaging and detailed electromyography study results, final diagnosis of cervical spondylosis could be made. Although coexistences and differential diagnosis difficulties of ALS and spondylotic myelopathy have been reported multiple times in literature, we think that our case constitutes a considerably smart sample of a patient illustrating detailed processes of differential diagnosis between these two entities. Via our case, we again point out to cervical spondylosis as an important differential diagnosis of ALS. Nonetheless, still a coincidental motor neuron disease in our case might not totally be excluded, therefore one more reevaluation after surgery has been planned. In conclusion, we emphasize the importance of complementary MRI and electro-

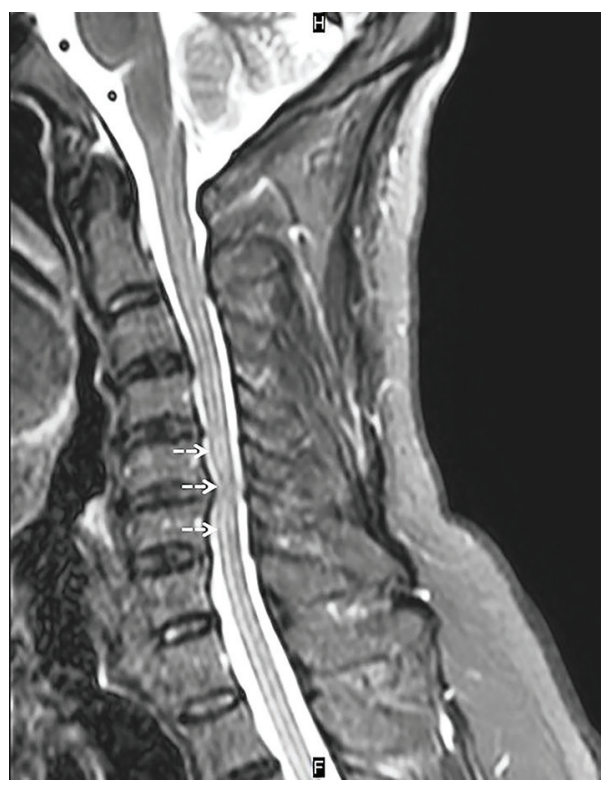

Figure 1. Cervical MRI (T2 sequence) showing central posterior protrusion at C5-6 level resulting in longitudinal myelitis extending from C4 to $\mathrm{C} 7$ segment. 
myography results in making the final diagnosis.

\section{Funding}

None.

\section{Conflicts of Interest}

There are no conflicts of interest.

\section{References}

1. Truffert A, Rosler KM, Magistris MR. Amyotrophic lateral sclerosis versus cervical spondylotic myelopathy: a study using transcranial magnetic stimulation with recordings from the trapezius and limb muscles. Clin Neurophysiol. 2000;111(6):1031-1038.

2. Ebara S, Yonenobu K, Fujiwara K, Yamashita K, Ono K. Myelopathy hand characterized by muscle wasting. A different type of myelopathy hand in patients with cervical spondylosis. Spine (Phila Pa 1976). 1988;13(7):785-791. 